BMJ

Open

Gastroenterology

\title{
Gastric microbiota in the functional dyspepsia patients treated with probiotic yogurt
}

\author{
Hirohiko Nakae, ${ }^{1}$ Ayumi Tsuda, ${ }^{2}$ Takashi Matsuoka, ${ }^{3,4}$ Tetsuya Mine, ${ }^{1}$ \\ Yasuhiro Koga, ${ }^{4}$
}

\begin{abstract}
Objective: To investigate the structure of the gastric microbiota in functional dyspepsia (FD) and its role in the pathophysiology.

Design: We compared the basic physiological properties of the gastric fluid (GF) and the structure of the microbiota in the GF of 44 healthy control $(\mathrm{HC})$ participants and 44 patients with FD. We then treated the patients with $\mathrm{FD}$ with a yogurt containing a probiotic strain of Lactobacillus gasseri OLL2716 (LG21 yogurt) and investigated the effects on the bacteriological parameters and symptoms to examine the relationship between them.
\end{abstract}

Results: The volume of GF recovered from the stomach after overnight fasting was greater in the patients with $\mathrm{FD}$ than in the $\mathrm{HCs}$, and decreased in the patients with $F D$ whose symptoms were improved by the LG21 yogurt treatment. An analysis using a terminal restriction fragment polymorphism method demonstrated that the overall structure of the bacterial community and the abundance of genus Prevotella in the GF of the patients in the FD group were significantly different from those in the $\mathrm{HC}$ group. In the patients with $F D$, this bacteriological change was restored by treatment with LG21 yogurt. A significant inverse correlation was found between the abundance of Prevotella and the severity of postprandial distresslike symptoms in patients with FD who received LG21 yogurt.

Conclusions: Significant dysbiosis was found in the GF microbiota of patients with FD and considered to be involved in the pathogenesis. The abundance of genus

'Department of Gastrointestinal Medicine, Tokai University School of Medicine, Isehara, Japan 2Department of General Medicine, Tokai University School of Medicine, Isehara Japan

${ }^{3}$ Koikeya Co., Ltd, ItabashiKu, Tokyo, Japan ${ }^{4}$ Laboratory for Infectious Diseases, Tokai University School of Medicine, Isehara, Japan

\section{Correspondence to} Dr Yasuhiro Koga; yasuhiro@is.icc.u-tokai.ac.jp
Prevotella in the GF may be used as a biomarker of the efficacy of the treatment of FD.

Trial registration number: UMINCTR000022026.

\section{INTRODUCTION}

Functional dyspepsia (FD) is defined as the presence of symptoms that are thought to originate in the gastroduodenal region, in the absence of any organic, systemic or metabolic disease that is likely to explain the symptoms. Accumulating evidence has revealed the heterogeneity of putative underlying mechanisms in FD. Thus, it is now

\section{Summary box}

What is already known about this subject? Although the pathophysiology of functional dyspepsia (FD) still remains unclear, delayed gastric emptying, impaired gastric accommodation, and visceral hypersensitivity have been suggested as major underlying mechanisms. However, no sufficient microbiological data have been available to explain the pathophysiology underlying FD.

What are the new findings?

Significant dysbiosis was found in the gastric fluid microbiota of patients with FD and considered to be involved in the pathogenesis. The dysbiosis was restored by treatment with the yogurt containing a probiotic lactobacillus strain. A significant inverse correlation was found between the abundance of genus Prevotella and the severity of postprandial distress-like syndromes in patients with FD who received a probiotic yogurt.

How might it impact on clinical practice in the foreseeable future?

These findings increase the chance of obtaining important information that may improve the diagnosis and treatment of FD from viewpoint of the gastric microbiota. The abundance of genus Prevotella in the gastric fluid may be used as a biomarker of the efficacy of the treatment of FD.

considered that this syndrome consists of at least two major subcategories such as epigastric pain syndrome and postprandial distress syndrome. ${ }^{1}$

Although the pathophysiology of FD remains unclear, delayed gastric emptying, impaired gastric accommodation after meals and visceral hypersensitivity have been suggested as underlying central mechanisms. ${ }^{2}$ Among those discordant gastric functions in FD, delayed emptying causes the long-term retention of ingested foods in the stomach, which may lead to a change in the gastric fluid (GF) microbiota. In addition, many patients with FD feel that their symptoms are 
related to the ingestion of food so that they can eat only small meals and do not tolerate fat; thus, they tend to consume carbohydrate-rich meals. ${ }^{3}{ }^{4}$ An alteration in the type of ingested foods is also suggested to affect the composition of the gastric microbiota. Moreover, those possible changes in the gastric microbiota may be involved in the pathogenesis and pathophysiology of FD, through the metabolic activity of the luminal microbiota, and the possibly the mucosa-associated microbiota, which may influence the host via the immune-microbial interaction. However, no sufficient microbiological data have been available to explain the pathophysiology of the symptoms or to validate the end points that may serve as biomarkers for the diagnosis and treatment of FD.

Probiotics are live microorganisms which confer a health benefit on the host when they are administered in adequate amounts. ${ }^{5}$ As the normalisation of the perturbed microbiota is one of the predominant mechanisms underlying the effects of probiotics, they seem to have potential in the treatment of the symptoms of patients with FD in whom the gastric microbiota is perturbed. ${ }^{6}$ Actually in our previous clinical trial involving patients with $\mathrm{FD}$, the administration of a probiotic yogurt containing Lactobacillus gasseri OLL2716 (LG21 yogurt) significantly improved the symptoms of postprandial distress; however, we did not analyse the gastric microbiota or elucidate the underlying mechanism.

The 16S rRNA (16S) gene sequence is now increasingly used in the analysis of microbiota because it can characterise the overall structure of the bacterial community and the bacterial composition using samples from different sites of the body. ${ }^{8}$ Among these $16 \mathrm{~S}$ genedependent methods, the terminal restriction fragment length polymorphism (T-RFLP) is quite efficient for the rapid assessment of the structure of the bacterial community because the skill of T-RFLP is relatively easy and it may work with numerous types of samples, including routine clinical samples. Moreover, T-RFLP can analyse the structure of the bacterial community, even in cases in which the bacterial count is small and the number of dominant bacterial species is limited. ${ }^{9}{ }^{10}$ Thus, we used the T-RFLP to characterise the gastric microbiota in the present study because the bacterial count and the composition of gastric microbiota were reported to be limited. ${ }^{11}$

To identify the bacteriological biomarkers of FD, we first compared the basic physiological properties of the GF and the microbiota structure in the GF of healthy control (HC) participants and patients with FD. We then treated the patients with FD with LG21 yogurt and investigated the effects of the yogurt on stomach-related items that have been identified as being FD specific in order to confirm that those items are really correlated with the symptoms. The LG21 yogurt treatment was administered because it had been found to positively change the symptoms in patients with FD in our previous study. ${ }^{7}$ The rationale for these steps was the assumption that if there was a significant correlation between a symptom and a stomach-related bacteriological parameter, we could increase the chance of obtaining important information that may help to improve the diagnosis and treatment of FD from the viewpoint of the gastric microbiota.

\section{METHODS}

\section{The participants}

Forty-four Japanese patients with FD at the age of 20 60 years who met the definition of the Rome III classification were enrolled from April 2014 to March 2016 (table 1). The exclusion criteria were the use of antimicrobials within the previous 3 months, the use of acid-suppressive drugs such as proton-pump inhibitors, serologically positive for anti-Helicobacter pylori antibodies, an allergy to lactic acid bacteria products and a history of gastrointestinal (GI) or hepatobiliary surgery. Twenty-four randomly selected patients also underwent a T-RFLP analysis. A total of 44 healthy volunteers at the age of 20-60 years were enrolled as HCs. The exclusion criteria were the same as those for patients with FD. Twenty-one HCs also underwent a T-RFLP analysis. The ethics committee of Tokai University Hospital approved the study (13R-324, 5 March 2014) and written informed consent was obtained from all of the participants. A clinical part of this study is registered in UMIN-CTR website (http://www.umin.ac.jp/ctr; Test 000022026).

\section{Study protocol}

All of the patients with FD were asked to consume $118 \mathrm{~g}$ of yogurt containing $10^{9}$ colony-forming units of LG21 (LG21 yogurt) every day for a 12-week-treatment period. The patients were informed that the end point of the study was to examine the influence of yogurt on 'the GF microbiota'. The compliance (the number of days for which yogurt was consumed) was evaluated through a diary written by the participant. The patients had clinical examinations before and after the yogurt treatment. In the morning on the day of examination, blood and GF were sampled from the participants after an overnight fast. For the sampling of GF, a nasogastric tube was inserted into the stomach through the nostril and then as much GF as possible was aspirated using a disposable syringe connected to the tube and then transferred into a test tube. The participants were then asked to fill out a frequency scale for the symptoms of GERD (FSSG) questionnaire while they were unaware that the symptoms were also analysed in the study. Finally, the participants underwent a urinary sucrose excretion test. The HC underwent the same clinical examinations as the patients with GF.

\section{The questionnaire}

The FSSG questionnaire was originally produced by Kusano $e t a l^{12}$ as a method of evaluating the symptoms of GERD and FD because the overlap of both conditions is quite common in Asian populations, including Japanese. ${ }^{13}$ This questionnaire is composed of 12 
Table 1 Demographic and clinical data

\begin{tabular}{|c|c|c|c|c|c|}
\hline & \multirow[b]{2}{*}{ HCs $(n=44)$} & \multicolumn{2}{|l|}{ FD $(n=44)$} & \multicolumn{2}{|l|}{ p Value } \\
\hline & & $\begin{array}{l}\text { Before LG21 } \\
\text { treatment }\end{array}$ & $\begin{array}{l}\text { After } \\
\text { LG21treatment }\end{array}$ & $\begin{array}{l}\text { HC vs FD } \\
\text { before }\end{array}$ & $\begin{array}{l}\text { FD before } \\
\text { vs FD after }\end{array}$ \\
\hline Age of years & $41.5(30.3-48.5)^{\star}$ & $42.5(34.5-50.3)$ & & 0.27 & \\
\hline Male/female & $30 / 14$ & $22 / 22$ & & 0.083 & \\
\hline \multicolumn{6}{|l|}{ FSSG scores } \\
\hline Epigastric pain-like & $1.0(0-2.0)$ & $12.0(7.8-15)$ & $4.0(3.0-6.3)$ & $<0.001$ & $<0.001$ \\
\hline Postprandial distress-like & $2.0(1.0-3.0)$ & $15.0(13.0-17.3)$ & $7.3(3.0-10.3)$ & $<0.001$ & $<0.001$ \\
\hline \multicolumn{6}{|l|}{ GF } \\
\hline Volume (mL) & $18.6 \pm 9.2 \dagger$ & $23.4 \pm 10.4$ & $19.7 \pm 9.5$ & 0.026 & 0.046 \\
\hline $\mathrm{pH}$ & $1.65(1.40-1.86)$ & $1.58(1.43-1.85)$ & $1.84(1.56-3.81)$ & 0.72 & 0.012 \\
\hline $\begin{array}{l}\text { Number of cultured bacteria } \\
\left(\log _{10} \mathrm{CFU} / \mathrm{mL}\right)\end{array}$ & $2.3(1.3-4.8)$ & $2.4(1.3-4.0)$ & $3.7(1.7-6.8)$ & 0.91 & 0.050 \\
\hline \multicolumn{6}{|l|}{ Serum } \\
\hline Pepsinogen I & 37.7 (29.4-44.0) & $33.3(27.5-41.9)$ & 34.7 (29.9-44.8) & 0.36 & 0.045 \\
\hline $\mathrm{PG} \mathrm{I} / \mathrm{II}$ ratio & $5.3(4.8-6.0)$ & $5.1(4.6-5.8)$ & $5.3(4.7-6.3)$ & 0.22 & 0.052 \\
\hline$\%$ urinary sucrose excretion $\left(\times 10^{3}\right)$ & $6.2(3.8-10)$ & $9.5(4.8-16)$ & $10(5.3-14)$ & 0.16 & 0.82 \\
\hline $\begin{array}{l}{ }^{*} \text { Median (IQR). } \\
\text { †Mean } \pm \text { SD. } \\
\text { CFU, colony-forming unit; FD, functional } \\
\text { LG21, Lactobacillus gasseri OLL2716; } P\end{array}$ & $\begin{array}{l}\text { pepsia; FSSG, } \mathrm{fl} \\
\mathrm{I} \text {, pepsinogens } \mathrm{I} /\end{array}$ & $y$ scale for the $s$ & ms of GERD; GF & ric fluid; $\mathrm{H}$ & ealthy control; \\
\hline
\end{tabular}

questions. Seven questions are related to gastrooesophageal reflux or epigastric pain-like symptoms, and these include, 'Do you get heartburn?', 'Do you sometimes subconsciously rub your chest with your hand?', 'Do you get heartburn after meals?', 'Do you have an unusual sensation in your throat?', 'Do some things get stuck when you swallow?', 'Do you get bitter liquid (acid) coming up into your throat?', and 'Do you get heartburn if you bend over?' Five questions are related to dysmotility-like dyspepsia or postprandial distress-like symptoms, and these include, 'Does your stomach get bloated?', 'Does your stomach ever feel heavy after a meal?', 'Do you ever feel sick after meals?', 'Do you feel full while eating meals?', and 'Do you burp a lot?'. Each of these questions was scored to indicate the frequency of symptoms as follows: never $=0$, occasionally $=1$, sometimes $=2$, often $=3$, and always $=4$.

\section{The analysis of the microbiota}

After sampling, $0.5 \mathrm{~mL}$ of GF was immediately spread over LB agar plates containing $5 \%$ horse serum after the sampling and then incubated at $37^{\circ} \mathrm{C}$ for 72 hours in $10 \% \mathrm{H}_{2}, 10 \% \mathrm{CO}_{2}$, and $80 \% \mathrm{~N}_{2}$. The remaining GF was immediately frozen and stored until the T-RFLP assay at $-50^{\circ} \mathrm{C}$. Bacterial DNAs were extracted from the GF using an Ultra Clean Soil DNA Isolation Kit (Mo Bio Laboratories, Carlsbad, California, USA) according to a manufacturer's instructions. The amplification of the GF $16 \mathrm{~S}$ rDNA, restriction enzyme digestion and the size fractionation of the terminal restriction fragments (T-RFs) were performed as previously reported. ${ }^{14}$ Briefly, a PCR was performed using the total GF DNA and the primers of $5^{\prime}$ HEX-labelled 516f (5'-TGCCAGCAGCCGCGGTA-3') and 1510r (5'-GGTTACGTTGTTACGACTT-3'). The resulting $16 \mathrm{~S}$ rDNA amplicons were treated with $2 \mathrm{U}$ of $B s I$
(New England BioLabs) for 1 hour and the digestive fragments were fractionated using an automated sequence analyser (ABI PRISM 310 Genetic Analyzer, Applied Biosystems, Foster City, California, USA) in GeneScan mode (the injection time was $20 \mathrm{~s}$ and the run time was $40 \mathrm{~min}$ ). The length and peak areas of the T-RFs were determined using the GeneScan software program v1.0 (Applied Biosystems). The sequencing of the major T-RFs that were detected using this T-RFLP method revealed that they corresponded to 24 phylogenic bacterial groups as reported elsewhere. ${ }^{14} \mathrm{~A}$ hierarchical clustering analysis of the individual T-RFs based on the unweighted pair group method with arithmetic mean (UPGMA) method and a principal coordinate analysis were performed using the Gene Maths software program v1.0 (Applied Biosystems). The difference between the overall bacterial community structures was examined using a permanent multivariate analysis of variance (PERMANOVA). The Bray-Curtis index was used to assess the interindividual diversity of the T-RFs among the $\mathrm{HC}$ and FD groups.

\section{The urinary sucrose excretion test}

Tests were performed after an overnight fasting. The participant drank $330 \mathrm{~mL}$ of water containing $50 \mathrm{~g}$ sucrose in the morning. Urine was collected for the following 4 hours in a plastic container to which $5 \mathrm{~mL}$ of $5 \%$ chlorhexidine gluconate was added. The volume voided was measured and aliquots were frozen at $-50^{\circ} \mathrm{C}$ until the assay. The urinary sucrose concentration was measured by the high performance liquid chromatography (HPLC). Although sucrose permeability is increased with gastroduodenal damage, this probe does not detect small intestinal damage because sucrose is rapidly degraded within the small intestine. ${ }^{15}$ 


\section{Statistical analysis}

Variables were reported as the medians and the IQR or the mean and SD according to their distribution. In a comparison between the $\mathrm{HC}$ and FD groups before treatment, the Student's t-test or the Mann-Whitney U test was used to perform the statistical analyses, after the examination of the normality using the Shapiro-Wilk test. In the FD group, the difference between before and after the treatment was statistically analysed using the paired t-test or the Wilcoxon signed-rank test according to the normality. A proportional odds logistic regression analysis was used to examine the influence of the determinants of each of the stomach-related parameters. Associations between the stomach-related parameters and the symptom scores were assessed by a rank test using Spearman's correlation coefficient. $p$ values of $<0.05$ were considered to indicate statistical significance.

\section{RESULTS}

\section{Demographic and clinical data}

The FSSG scores for epigastric pain-like and postprandial distress-like symptoms were far greater in the FD before LG21 treatment patients than in the HCs $(\mathrm{p}<0.001)$, while no difference was found between these two groups with regard to age or gender (table 1). The LG21 yogurt treatment significantly ameliorated the both of these symptoms in patients with FD $(p<0.001)$. Among the clinical stomach-related parameters, only the GF volume was significantly greater in the pretreatment patients with FD than in the HCs $(p=0.026)$. Furthermore, post-treatment GF volume was also significantly lower than the pretreatment GF volume $(p=0.046)$ in the patients with FD. In patients with FD, the $\mathrm{pH}$ value, the number of cultured bacteria, and the pepsinogen I (PGI) level were significantly increased after the LG21 treatment. No significant differences were found among these three groups with regard to the $\%$ urinary sucrose excretion, which represents the permeability of the gastric mucosa.

\section{Bacterial community}

The overall bacterial community structure of the GF microbiota was analysed using the T-RFLP method (figure 1). A cluster dendrogram representing the individual microbiota (A) showed a large distinct cluster that was composed of 12 patients with FD before LG21 treatment (red circles). A principal coordinate analysis (B) also demonstrated that the samples from the pretreatment FD group aggregated to form a cluster that was definitely separated from a cluster of the HC group ( $p=0.001$ by PERMANOVA test). However, the samples from the $\mathrm{HC}$ and the post-treatment $\mathrm{FD}$ groups appeared to form a common cluster $(p=0.149$ by PERMANOVA test). The average interindividual diversity of the bacterial communities (C) was significantly lower in the pretreatment FD group than in the HC group $(p<0.001)$. The treatment of patients with FD with LG21 yogurt tended to increase the diversity of the bacterial community. Taken together, these results indicated that the bacterial community of the pretreatment FD group was distinct from that in the HC group in terms of its structure and diversity. However, LG21 yogurt treatment restored this dysbiosis in the patients with FD to the extent that the structure and diversity were almost normal.

\section{Comparison of bacterial composition}

Next, the microbiota composition was analysed by comparing the relative abundance of eight major T-RFs (table 2). The abundance of Prevotella was significantly lower in the pretreatment patients with FD than in the HCs $(p=0.04)$. In addition, LG21 treatment significantly increased the abundance of Prevotella $(\mathrm{p}=0.001)$, suggesting a correlation of this bacterial genus with the symptoms. The independence of the change in the abundance of Prevotella from the other stomach-related parameters was supported by a logistic regression analysis. The relative abundance of Bifidobacterium, as well as Clostridium clusters IX, XI, and XVIII, was significantly greater in the pretreated patients with FD than in the HCs, while Bifidobacterium and Clostridium cluster XI were significantly less abundant in patients with FD after treatment than they had been before treatment; however, the values were too small to analyse further.

\section{Correlation between the stomach-related parameters and symptoms}

We finally tried to find an objective biomarker that was correlated with the symptoms of FD who underwent LG21 yogurt treatment. As a result, five stomach-related parameters were revealed to have significantly changed after the LG21 treatment (table 3). Among them, only the abundance of Prevotella was significantly correlated with the postprandial distress-like symptoms by Spearman's test $(\mathrm{r}=0.52, \mathrm{p}=0.009)$. The relationship between these values in each participant is shown in figure 2.

\section{DISCUSSION}

The present study focused on the analysis of microbiota in the stomach and investigated its role in the pathogenesis underlying FD. Among the stomach-related clinical parameters that were assessed in this study, the volume of GF appeared to be closely associated with the pathogenesis of FD, because the volume was greater in the patients with FD than in HC participants, and the volume was decreased in the patients with FD whose symptoms were improved by LG21 yogurt treatment. The GF volume, the fluid volume recovered from the stomach after overnight fasting, has been used to roughly assess the efficiency of gastric emptying. ${ }^{16}$ Thus, the increased GF volume in the patients with FD is thought to suggest the disturbance or delay of gastric emptying. Delayed gastric emptying is traditionally considered to be one of the major pathophysiological 
A

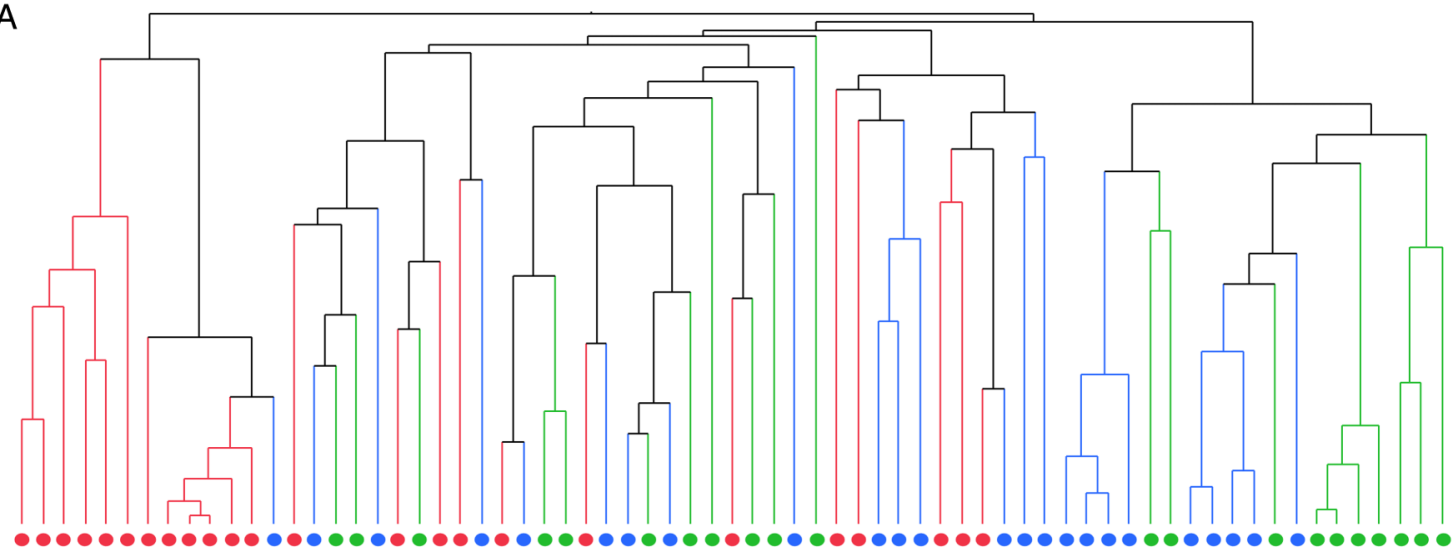

B

C
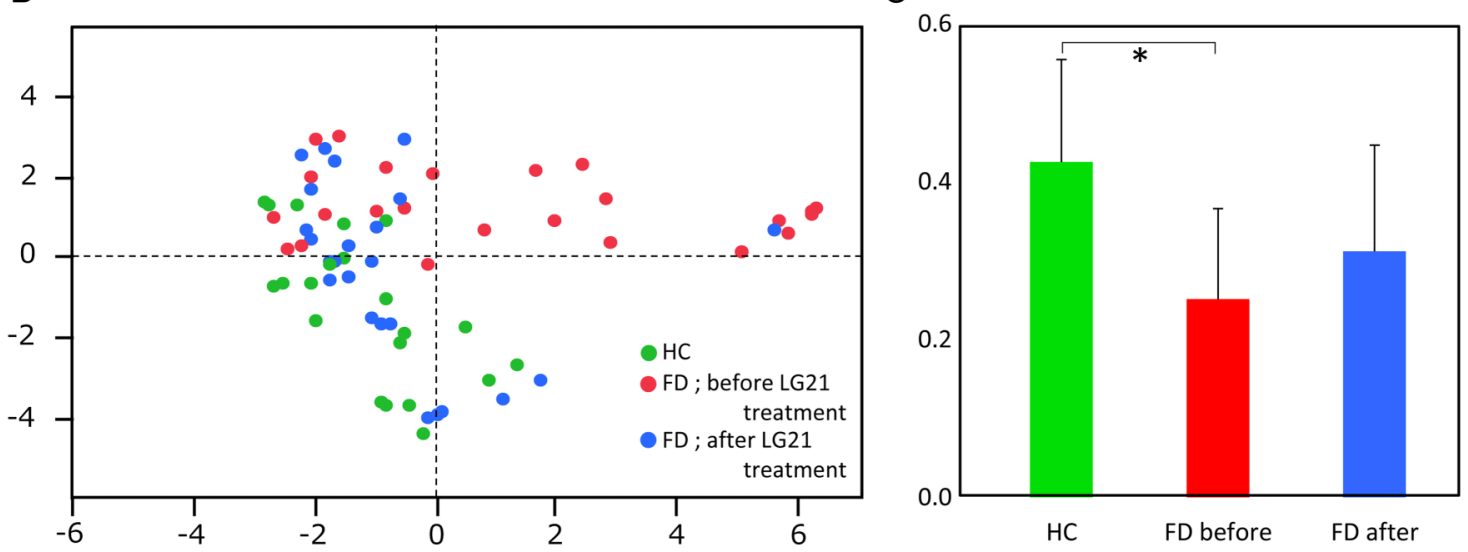

Figure 1 Analysis of bacterial community structure in GF. The overall bacterial community structure of the GF microbiota analysed by T-RFLP method was shown by cluster dendrogram (A), principal coordinate analysis ((B) PCo1 $=24.7 \%$,

PCo2 $=16.0 \%$ ) and Bray-Curtis index (C). Green, red, and blue symbols represent the HC, FD before LG21 treatment, and FD after LG21 treatment participants, respectively. Columns and bars in (C) represent the mean and SD, respectively. FD, functional dyspepsia; GF, gastric fluid; HC, healthy control; LG21, Lactobacillus gasseri OLL2716; T-RFLP, terminal restriction fragment length polymorphism. ${ }^{*} \mathrm{p}<0.001$.

Table 2 Relative abundance of T-RFs among the T-RFLP electropherogram

\begin{tabular}{|c|c|c|c|c|c|}
\hline \multirow[b]{2}{*}{ T-RFs corresponding to } & \multirow[b]{2}{*}{ HCs $(n=21)$} & \multicolumn{2}{|l|}{ FD $(n=24)$} & \multicolumn{2}{|l|}{ p Value } \\
\hline & & $\begin{array}{l}\text { Before LG21 } \\
\text { treatment }\end{array}$ & $\begin{array}{l}\text { After LG21 } \\
\text { treatment }\end{array}$ & $\begin{array}{l}\text { HC vs FD } \\
\text { before }\end{array}$ & $\begin{array}{l}\text { FD before vs } \\
\text { FD after }\end{array}$ \\
\hline Bifidobacterium & $0.0(0.0-0.9)^{*}$ & $2.5(0.2-5.4)$ & $0.4(0.2-0.7)$ & $<0.001$ & 0.003 \\
\hline Order Lactobacillales & $34.4(22.3-46.2)$ & $28.0(17.7-35.7)$ & $21.0(11.7-39.2)$ & 0.24 & 0.12 \\
\hline Prevotella & $37.4(18.8-52.1)$ & $28.3(19.1-26.1)$ & $39.8(25.6-52.1)$ & $0.04 \dagger$ & $0.001 \ddagger$ \\
\hline Clostridium cluster IV & $0.7(0.0-2.1)$ & $1.0(0.4-2.8)$ & $1.1(0.6-1.8)$ & 0.12 & 0.78 \\
\hline Clostridium subcluster XIVa & $10.8 \pm 5.4$ & $12.3 \pm 4.6$ & $10.2 \pm 3.9$ & 0.33 & 0.09 \\
\hline Clostridium cluster IX & $3.5(1.9-4.8)$ & $4.3(3.3-7.1)$ & $4.3(2.4-6.6)$ & 0.02 & 0.32 \\
\hline Clostridium cluster XI & $0.0(0.0-0.0)$ & $0.4(0.0-1.1)$ & $0.0(0.0-0.0)$ & $<0.001$ & 0.007 \\
\hline Clostridium cluster XVIII & $0.0(0.0-1.2)$ & $1.1(0.6-1.5)$ & $1.8(0.8-2.5)$ & 0.004 & 0.26 \\
\hline Others & $6.9(5.3-8.5)$ & $13.2(8.9-17.9)$ & $6.2(4.2-8.8)$ & $<0.001$ & 0.002 \\
\hline
\end{tabular}

*Median (IQR).

TOR (FD before vs $\mathrm{HC}) 1.27,95 \% \mathrm{Cl} 1.01$ to $1.59, \mathrm{p}=0.04$ by a logistic regression analysis in which the volume and the $\mathrm{pH}$ and the abundance of Prevotella were included in the independent variables.

fOR (FD after vs FD before) $1.76,95 \% \mathrm{Cl} 1.28$ to $2.42, \mathrm{p}<0.001$ by the same analysis as that noted above.

FD, functional dyspepsia; HC, healthy control; LG21, Lactobacillus gasseri OLL2716; T-RFLP, terminal restriction fragment length polymorphism. 
Table 3 Correlation between the changes in the objective parameters and the symptom scores in the patients with FD treated with LG21

\begin{tabular}{|c|c|c|c|c|}
\hline \multirow{3}{*}{$\begin{array}{l}\text { Stomach-related } \\
\text { parameters }\end{array}$} & \multicolumn{4}{|c|}{ Symptoms } \\
\hline & \multicolumn{2}{|c|}{$\begin{array}{l}\Delta \text { (epigastric } \\
\text { pain-like) }{ }^{\star}\end{array}$} & \multicolumn{2}{|c|}{$\begin{array}{l}\Delta \text { (postprandial } \\
\text { distress-like)* }\end{array}$} \\
\hline & $\bar{r}$ & p Value & $\overline{\mathbf{r}}$ & p Value \\
\hline \multicolumn{5}{|l|}{ GF } \\
\hline$\Delta$ (volume) & $0.11 \dagger$ & 0.49 & $0.07 \dagger$ & 0.65 \\
\hline$\Delta(\mathrm{pH})$ & $0.06 \ddagger$ & 0.69 & $0.04 \ddagger$ & 0.82 \\
\hline $\begin{array}{l}\Delta \text { (no. of cultured } \\
\text { bacteria) }\end{array}$ & $0.01 \ddagger$ & 0.93 & $0.05 \ddagger$ & 0.76 \\
\hline $\begin{array}{l}\Delta \text { (abundance of } \\
\text { Prevotella) }\end{array}$ & $0.38 \ddagger$ & 0.07 & $0.52 \ddagger$ & 0.009 \\
\hline$\Delta$ (serum PGI) & $0.07 \ddagger$ & 0.67 & $0.07 \ddagger$ & 0.64 \\
\hline \multicolumn{5}{|c|}{$\begin{array}{l}\text { * } \Delta \text { score (before LG21 treatment-after LG21 treatment). } \\
\text { †Pearson's product-moment correlation coefficient. } \\
\text { łSpearman’s rank correlation coefficient. } \\
\text { FD, functional dyspepsia; GF, gastric fluid; LG21, Lactobacillus } \\
\text { gasseri OLL2716. }\end{array}$} \\
\hline
\end{tabular}

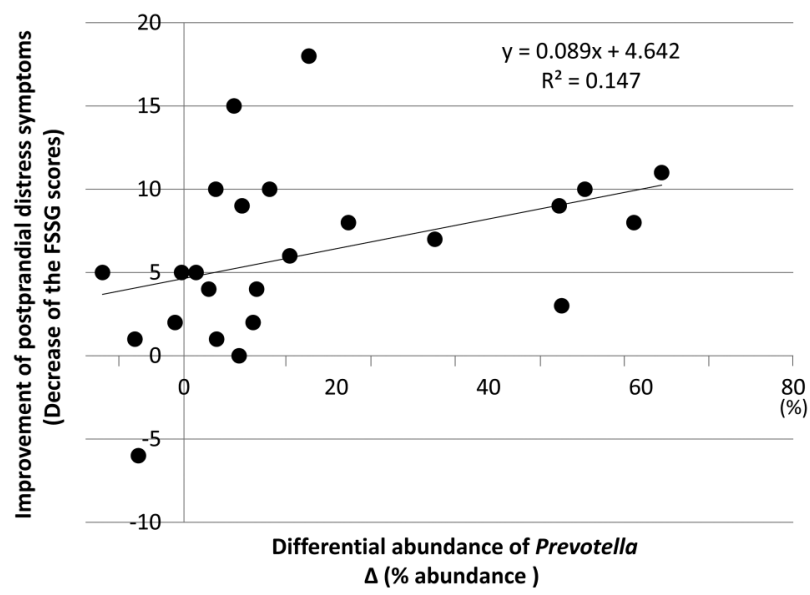

Figure 2 Relationship between differential abundance of Prevotella and improvement of postprandial distress-like symptoms. The increase in the abundance of Prevotella (horizontal axis) and the decrease in the FSSG scores for postprandial distress-like symptoms (vertical axis) of each patient with FD after LG21 treatment were plotted by a filled circle to evaluate Spearman's correlation coefficient. FD, functional dyspepsia; FSSG, frequency scale for the symptoms of GERD; LG21, Lactobacillus gasseri OLL2716.

mechanisms underlying FD symptoms, especially postprandial distress syndromes. ${ }^{2}$ Thus, the improvement of this symptom by LG21 yogurt treatment would validate the effectiveness of the use of probiotic yogurt in the treatment of FD because the improvement was accompanied by a decrease in the GF volume, which is considered to be an objective biomarker in FD. While well-regulated GI transit is one of the common beneficial effects exerted by probiotics, ${ }^{6}$ the mechanism through which LG21 yogurt improves delayed gastric emptying remains to be clarified. The suppression of gastric acid secretion shown by a higher $\mathrm{pH}$ value after LG21 treatment (table 1) might be involved in the improvement because, conversely, it was reported that acid challenge in the stomach aggravated the delay of gastric emptying in an experimental system using rats. ${ }^{16}$

Improvement in the symptoms by LG21 yogurt might have bias because neither placebo nor blind treatment was performed in the present study. Therefore, in this study, the participants were unaware that the symptoms were also analysed in order to reduce such bias. Moreover, the efficacy of LG21 yogurt in the treatment of postprandial distress symptoms was already demonstrated in a randomised double-blind controlled trial of H. pylori-infected participants. ${ }^{7}$ Nevertheless, the placebo treatment might have been needed in the present study too.

Although there is accumulating evidence to suggest that changes to the gut microbiota are involved in the pathophysiology of irritable bowel syndrome (IBS), a functional GI disorder (FGID) originating from the intestine, little is known about the gastric microbiota or its role in the pathophysiology of FD, an FGID that originates from the stomach and duodenum. ${ }^{8}$ However, in the present study, the use of the 16S-based T-RFLP method clearly demonstrated a significant difference in the overall bacterial community structure in the GF microbiota of the FD and HC groups. In line with the changes that were observed in the overall structure analysis, the bacterial composition analysis also showed the significant differences in the abundances of Prevotella (the abundance of which was lower in the patients with FD than in the HCs) and Bifidobacterium/Clostridium (the abundance of which were higher in the patients with FD than in the $\mathrm{HCs}$ ). One possible mechanism underlying this dysbiosis is that the long-term retention of gastric contents due to the delay of emptying, suggested in the present study, might change gastric environmental factors such as partial oxygen supply, acidity and mucus constitution, thereby influencing the bacterial colonisation in the stomach. The decrease in the interindividual diversity of the GF microbiota in the FD also suggests the occurrence of environmental changes that limit the ecological capacity for the bacteria in the stomach of these patients.

Prevotella and Bifidobacterium/Clostridium are known to predominantly inhabit the stomach and gut, respectively. ${ }^{17}$ In our previous study using GF samples from 45 healthy participants, the abundance of genus Prevotella was the second greatest among all of the genera, and accounted for $\sim 15 \%$ of the bacteria in each of the samples from the participants. However, the abundance of Bifidobacterium/Clostridium was $<0.1 \%$. In the fecal samples, on the other hand, few Prevotella were found. Furthermore, Bifidobacterium and Clostridium were the most abundant $(\sim 20 \%)$ and fourth most abundant $(\sim 7 \%)$ genera, respectively. ${ }^{18}$ Thus, changes in the bacterial composition in FD, such as a relative decrease in the abundance of Prevotella and the substantial appearance of Bifidobacterium/Clostridium in the GF, might be 
caused by the back transfer of intestinal contents, including bacteria, to the stomach. Indeed proximal small intestinal motor abnormalities and disturbed gastric motility are observed in patients with FD. An ambulatory duodenojejunal manometry study demonstrated that non-propagated and retrogradely propagated phase III activity frequently occurred in patients with FD, which may induce or accelerate the reflux of proximal small intestinal bacteria to the stomach. ${ }^{19}$ According to a clinical report, ${ }^{20}>60 \%$ of Japanese patients with FD have overlapping IBS in which small intestinal bacterial overgrowth (SIBO) has been reported as an aetiologic factor. ${ }^{8}$ Thus, many of our patients with FD could have had SIBO, which can cause the migration of large numbers of intestinal bacteria (such as Bifidobacterium) to the stomach through the reflux of the duodenojejunal contents. In addition, the disturbance of gastric emptying in patients with FD may result in these intestinal bacteria remaining in the stomach for prolonged periods of time because deranged antroduodenal motor activity fail to expel them.

In the microbiological studies of FGID that have been performed thus far, little attention has been paid to the correlation between changes in the microbiota and patients' symptoms. Thus, the cause-effect relationship between them has largely remained unclear. In the present study, we found a significant inverse correlation between the abundance of Prevotella and the severity of postprandial distress-like symptoms in patients with FD who underwent probiotic yogurt treatment. This suggests that a reduction of this genus in the GF is closely related to the triggering mechanisms of the symptoms. In addition, the abundance of Prevotella may be used as a biomarker of the efficacy of FD treatment. Bacteria of the genus Prevotella are the predominant inhabitants of the oral cavity and stomach, and are also bile sensitive. ${ }^{21}$ Although there is a possibility that Prevotella or its metabolites protect the stomach from the pathophysiological events that induce symptoms, there is little evidence to support this. As mentioned above, the reflux of the duodenojejunal contents was considered to be an important pathophysiological mechanism in the present study. This event would bring bile as well as intestinal bacteria to the stomach. Since Prevotella is vulnerable to bile acids, its number would be reduced to a much greater extent than the other bacteria when such contents were brought to the stomach. Moreover, the bile acids and pancreatic juice in the contents may irritate the gastric mucosa leading to the aggravation of symptoms. Thus, in this hypothesis, a decrease in the abundance of Prevotella in the GF closely reflects the enhancement of the reflux of bowel contents, which is assumed to trigger the symptoms of FD.

In the present study, intervention using a yogurt containing LG21, a probiotic lactobacillus strain, almost restored the dysbiotic microbiota in patients with FD to the levels that are found in HC participants. Since this treatment also improved their symptoms, it is thought that the attenuation of the reflux and the efficient disposal of the refluxed contents are the mechanisms by which the LG21 exerted its effect. Indeed, the numbers of Prevotella and Bifidobacterium/Clostridium increased and became minimal, respectively, after the treatment. Considering that lactobacilli are well known to adsorb bile acids, ${ }^{22}$ the lactobacilli in the yogurt may remove bile acids in the GF and thus may be partly responsible for the restoration of Prevotella. As the pharmacological treatments that are currently available to date for the management of FD have only been shown to be of limited efficacy, the development of therapeutic approaches towards the unknown pathophysiological disturbances appears to be a promising direction for future treatment. In this respect, the administration of probiotics to correct gastric dysbiosis seems to be logical and may be useful in the treatment of FD.

Contributors HN and AT recruited the patients with FD and healthy participants, had interview with them, and collected the clinical samples. TMa performed bacteriological and genetic analyses of the samples. TMi was involved in the study concept. YK was involved in the study concept and design, data interpretation, and drafting the manuscript.

Funding This work was financially supported by Research Fund of Tokai University School of Medicine.

Competing interests None declared.

Ethics approval The Ethics Committee of Tokai University Hospital.

Provenance and peer review Not commissioned; externally peer reviewed.

Data sharing statement No additional data are available.

Open Access This is an Open Access article distributed in accordance with the Creative Commons Attribution Non Commercial (CC BY-NC 4.0) license, which permits others to distribute, remix, adapt, build upon this work noncommercially, and license their derivative works on different terms, provided the original work is properly cited and the use is non-commercial. See: http:// creativecommons.org/licenses/by-nc/4.0/

\section{REFERENCES}

1. Tack J, Talley NJ, Camilleri M, et al. Functional gastroduodenal disorders. Gastroenterology 2006;130:1466-79.

2. Tack J, Bisschops R, Sarnelli G, et al. Pathophysiology and treatment of functional dyspepsia. Gastroenterology 2004;127:1239-55.

3. Pilichiewicz AN, Feltrin KL, Horowitz M, et al. Functional dyspepsia is associated with a greater symptomatic response to fat but not carbohydrate, increased fasting and postprandial CCK, and diminished PYY. Am J Gastroenterol 2008;103:2613-23.

4. Pilichiewicz AN, Horowitz M, Holtmann GJ, et al. Relationship between symptoms and dietary patterns in patients with functional dyspepsia. Clin Gastroenterol Hepatol 2009;7:317-22.

5. FAO of the UN and WHO. Health and nutritional properties of probiotics in food including powder milk with live lactic acid bacteria. WHO, 2001. http://www.who.int/foodsafety/publications/fs_ management/en/probiotics.pdf

6. Hill C, Guarner F, Reid G, et al. Expert consensus document. The international scientific association for probiotics and prebiotics consensus statement on the scope and appropriate use of the term probiotic. Nat Rev Gastroenterol Hepatol 2014;11:506-14.

7. Takagi A, Yanagi $\mathrm{H}$, Ozawa $\mathrm{H}$, et al. Effect of Lactobacillus gasseri OLL2716 on Helicobacter pylori-associated dyspepsia: a multicenter randomized double-blind controlled trial. Gastroenterol Res Pract 2016;2016:7490452.

8. Simrén M, Barbara G, Flint HJ, et al., Rome Foundation Committee. Intestinal microbiota in functional bowel disorders: a Rome foundation report. Gut 2013;62:159-76.

9. Liu WT, Marsh TL, Cheng $\mathrm{H}$, et al. Characterization of microbial diversity by determining terminal restriction fragment length 
polymorphisms of genes encoding 16S rRNA. Appl Environ Microbiol 1997;63:4516-22.

10. Nagashima K, Hisada T, Sato M, et al. Application of new primer-enzyme combinations to terminal restriction fragment length polymorphism profiling of bacterial populations in human feces. Appl Environ Microbiol 2003;69:1251-62.

11. Bik EM, Eckburg PB, Gill SR, et al. Molecular analysis of the bacterial microbiota in the human stomach. Proc Natl Acad Sci USA 2006;103:732-7.

12. Kusano M, Shimoyama $\mathrm{Y}$, Sugimoto $\mathrm{S}$, et al. Development and evaluation of FSSG: frequency scale for the symptoms of GERD. J Gastroenterol 2004;39:888-91.

13. Miwa H, Ghoshal UC, Gonlachanvit S, et al. Asian consensus report on functional dyspepsia. J Neurogastroenterol Motil 2012;18:150-68

14. Nagashima K, Mochizuki J, Hisada T, et al. Phylogenetic analysis of 16S ribosomal RNA gene sequences from human fecal microbiota and improved utility of terminal restriction fragment length polymorphism profiling. Bioscience Microflora 2006;25:99-107.

15. Meddings JB, Sutherland LR, Byles NI, et al. Sucrose: a novel permeability marker for gastroduodenal disease. Gastroenterology 1993:104:1619-26.
16. Holzer $\mathrm{P}$, Painsipp $\mathrm{E}$, Jocic $\mathrm{M}$, et al. Acid challenge delays gastric pressure adaptation, blocks gastric emptying and stimulates gastric fluid secretion in the rat. Neurogastroenterol Motil 2003;15:45-55.

17. The Human Microbiome Project Consortium. Structure, function and diversity of the healthy human microbiome. Nature 2012;486:207-14.

18. Tsuda A, Suda W, Morita $\mathrm{H}$, et al. Influence of proton-pump inhibitors on the luminal microbiota in the gastrointestinal tract. Clin Trans Gastroenterol 2015;6:e89.

19. Jebbink HJ, vanBerge-Henegouwen GP, Akkermans LM, et al. Small intestinal motor abnormalities in patients with functional dyspepsia demonstrated by ambulatory manometry. Gut 1996;38:694-700.

20. Hori K, Matsumoto T, Miwa H. Analysis of the gastrointestinal symptoms of uninvestigated dyspepsia and irritable bowel syndrome. Gut Liver 2009;3:192-6.

21. Paster BJ, Dewhirst FE, Olsen I. Phylogeny of Bacteroides, Prevotella, and Porphyromonas spp. and related bacteria. J Bacterio 1994:176:725-32.

22. Midtvedt T, Norman A. Adsorption of bile acids to intestinal microorganisms. Acta Path Microbiol Scand 1972;80:202-10. 